\title{
Elemental abundance study of the CP star HD 206653
}

\author{
J. F. Albacete-Colombo ${ }^{1}$, Z. López-García ${ }^{2,3,4}$, H. Levato ${ }^{2,3}$, S. M. Malaroda ${ }^{2,5}$, and M. Grosso ${ }^{2}$ \\ ${ }^{1}$ Facultad de Ciencias Astronómicas y Geofísicas, Universidad Nacional de La Plata, Paseo del Bosque S/N, \\ 1900 La Plata, Argentina \\ 2 Complejo Astronómico El Leoncito, C.C 467, 5400 San Juan, Argentina \\ ${ }^{3}$ Member of the Carrera del Investigador Científico, CONICET, Consejo Nacional de Investigaciones Científicas y Técnicas \\ de la República Argentina \\ ${ }^{4}$ Facultad de Ciencias Exactas, Físicas y Naturales, Universidad Nacional de San Juan, Argentina \\ 5 Comisión de Investigaciones Científicas de la Provincia de Buenos Aires, Argentina
}

Received 30 October 2001 / Accepted 11 April 2002

\begin{abstract}
An analysis of the abundances of the Silicon star HD 206653 is presented using an ATLAS9 model atmosphere and observational material taken with a REOSC echelle spectrograph attached to the Jorge Sahade $2.15 \mathrm{~m}$ telescope at CASLEO. The light elements are solar or deficient except silicon which is overabundant by a factor of 5. The iron peak elements are all overabundant by factors between 10 and 50. Sr and Y are around 1000 times the solar values. Among the Rare Earths only Ce and $\mathrm{Eu}$ are identified; both are overabundant by large factors.
\end{abstract}

Key words. stars: chemically peculiar - stars: abundances - stars: individual: HD 206653

\section{Introduction}

This research is part of our current program for deriving elemental abundances among $\mathrm{CP}$ stars. For this paper we have selected HD 206653. This star is also known as BC Ind $(V=7.2)$. It has been classified in the list of Bidelman \& Mac Connell (1973) as an Ap star of the silicon group. Differential $u v b y$ photometry has been carried out by Hensberge et al. (1977), and the results showed that the observations were well represented by a period of $1.788 \pm 0.005$; they also found that the $b-y$ color index varies slightly while $m_{1}$ remains constant. The peak-to-peak variation is larger in the $u$ filter $(1.2 \mathrm{mag})$ while in the other filters the peak-to-peak variations are not larger than 0.4 mag. Levato et al. (1996) estimated a radial velocity of $-0.5 \mathrm{~km} \mathrm{~s}^{-1}$ and a value for the projected rotational velocity of $\leq 30 \mathrm{~km} \mathrm{~s}^{-1}$. Table 1 presents some relevant observational data for HD 206653. The $U B V$ data and Strömgren photometry were taken from the CDS database. The latter values at the CDS were derived from Hauck \& Mermilliod (1980). The parallax is the Hipparcos' value (Perrymann et al. 1997).

Strömgren photometry has been also published by Maitzen et al. (2000) and there are $\Delta a$ measures by Maitzen \& Vogt (1983).

\section{Observational material and line identifications}

Spectra of HD 206653 were obtained by SM and MG with the Jorge Sahade 2.15-m telescope at Complejo Astronómico

Send offprint requests to: $\mathrm{H}$. Levato,

e-mail: hlevato@casleo.gov.ar
Table 1. Observational data for HD 206653.

\begin{tabular}{lc}
\hline \hline Parameter & value \\
\hline$B-V$ & -.07 \\
$U-B$ & -0.24 \\
$v$ & 7.191 \\
$b-y$ & -0.031 \\
$m_{1}$ & 0.137 \\
$c_{1}$ & 0.549 \\
$\beta$ & 2.740 \\
$R V\left[\mathrm{~km} \mathrm{~s}^{-1}\right]$ & -0.5 \\
parallax [0.'001] & 4.14 \\
\hline
\end{tabular}

el Leoncito (CASLEO) equipped with a REOSC echelle spectrograph $^{1}$ and a TEK $1024 \times 1024$ CCD detector. Two spectra were obtained covering the visual range $\lambda \lambda 3640-5400$, and two covering the red region $\lambda \lambda 6200-8450$. For the latter, those regions with telluric contamination were not measured. The REOSC spectrograph used gratings as cross dispersers. We have used one grating with 400 lines $\mathrm{mm}^{-1}$ and another with 315 lines $\mathrm{mm}^{-1}$ for the blue and red region respectively. The $S / N$ ratio of the spectra is around 100 and the blue spectra were taken in two consecutive nights (HJD 2451031.736 and 2451032.789) while each red spectrum was secured at HJD 2451416.652 and 2451417.683 respectively. Using the ephemeris derived by Hensberge et al. (1977), the HJD indicated correspond to phases $0.725,0.314,0.003$ and 0.579 in

${ }^{1}$ On loan from the Institute d'Astrophysique de Liège. 
increasing order of HJD. There is no more than $20 \%$ difference among the equivalent width measurements of the same lines in different spectra. The spectra were reduced by MG, using $\mathrm{IRAF}^{2}$ standard procedures for echelle spectra, and they were normalized order by order with the splot task of the same package. The resolution of the spectra is $0.17 \AA / \mathrm{px}$ in the blue and $0.21 \AA / p x$ in the red. Extensive description and tests of observational material obtained with the same equipment, and astrophysical results derived from them, have been published by Pintado \& Adelman (1996), Adelman \& Pintado (1997), Pintado et al. (1998) and more recently by Z. López García et al. (2001). The equivalent widths were measured by fitting Gaussian profiles through the stellar metal lines using the same task. The equivalent width scale of these spectrograms has been proved to be marginally larger than those for Kitt Peak National Observatory (KPNO) coude feed CCD and Dominion Astrophysical Observatory (DAO) Reticon spectrograms. See details in Pintado \& Adelman (1996) and in Adelman \& Pintado (2000). Line identification of HD 206653 has been carried out by Albacete Colombo et al. (2001), in preparation. The stellar lines were identified using A Multiplet Table of Astrophysical Interest (Moore 1945) and Wavelengths and Transition Probabilities for Atoms and Atomic Ions, Part 1 (Reader \& Corliss 1980) as well as the more specialized references for S II: Pettersson (1983), Ti II: Huldt et al. (1982), Mn II: (Iglesias \& Velasco 1964), and Fe II: (Johansson 1978). Lines of $\mathrm{H}_{\text {I, }} \mathrm{C}_{\text {II, }} \mathrm{Mg}_{\text {I, }} \mathrm{Mg}_{\text {III }} \mathrm{Al}_{\mathrm{I}}, \mathrm{Si}$ II, Si III, S II, Ca II, Sc II, Ti II, Cr II, Mn II, Fe II, Fe III, Ni II, Sr II, Y II, Zr II, Ce II, and Eu II are definitely present in $\mathrm{HD} 206653$. Lines of $\mathrm{Ba}$ II and $\mathrm{Hg}$ II are probable present.

\section{Atmospheric parameters}

The determination of effective temperatures for Ap stars is a difficult problem. North \& Kroll (1989) estimated the effective temperature for HD 206653 using two different methods. First they used the calibration of the Geneva photometry provided by Lanz (1987) and they obtained $T_{\text {eff }}=12350 \mathrm{~K}$. Second, they used the calibration by North \& Nicolet (1990) of the Geneva $Y / X$ diagram, valid for stars whose $[\mathrm{M} / \mathrm{H}]$ are equal to 1.0 , which is more realistic than the solar composition models used by Lanz, and they obtained $T_{\text {eff }}=13230 \mathrm{~K}$. From these previous temperature estimations, North \& Kroll (1989) estimated surface gravities for CP stars and obtained $\log g=$ $3.52 \pm 0.10$ and $3.73 \pm 0.10$ respectively for each temperature value. Another estimation of the effective temeprature was done by Glagolevskij (1994), who used the Shallis-Blackwell method starting with the total flux emitted by HD 206653, and he found $12600 \mathrm{~K}$.

For consistency with previous papers published by our group (see Z. López García et al. 2001), we used $u v b y \beta$ mean colors from Hauck \& Mermilliod (1980) with the calibration of Napiwotzki et al. (1993) to obtain an initial estimate of

\footnotetext{
2 IRAF is distributed by the National Optical Astronomical Observatories which is operated by the Association of Universities for Research in Astronomy, Inc., under a cooperative agreement with the National Science Foundation.
}

the atmospheric parameters of HD 206653. The result was $T_{\text {eff }}=13370 \mathrm{~K}$ and $\log g=3.95$. As there are no spectrophotometric measures for this star, we used the correction given in Adelman \& Rayle (2000) to estimate a spectrophotometric temperature using $u v b y \beta$ photometry and we found $T_{\text {eff }}=14535 \mathrm{~K}, \log g=3.86$. We finally adopted these values. Errors in these two parameters are around $200 \mathrm{~K}$ for the effective temperature and 0.2 dex in $\log g$ according to the discussion by Adelman \& Rayle (2000). These values agree with the errors discussed by Napiwotzki et al. (1993) for normal star from middle B to early F. With the values of $\log g$ and effective temperature determined as explained above we have computed a model atmosphere using Kurucz's ATLAS9 code with $[\mathrm{M} / \mathrm{H}]=1$, which is characteristic of the Silicon stars.

\section{Abundance analyses}

We determined the metal abundances from the average equivalent widths of the two blue spectra and the average of the two red spectra, using the WIDTH9 code (Kurucz 1992). The adopted metal line damping constants were the default semiclassical approximations except for those of neutral and singlyionized $\mathrm{Ca}-\mathrm{Ni}$ lines whose values are based on the data of Kurucz \& Bell (1995). For lines of C II, multiplet 6, and Mg II, multiplet 4, adopted values for the Stark broadening were based on data of Sahal-Brechot (1969) and for Si II and Ca II, the damping constants are those of Lanz et al. (1988) and Chapelle \& Sahal-Brechot (1970) respectively. We prefer this choice of $g f$ values to the VALD database values (Piskunov 1996) to assure homogeneity with our previous work. For calculating the microturbulent velocity we have used the standard method. We calculated abundances from FeII lines for HD 206653 for a range of possible microturbulent velocities. To determine the final value, we looked for the conditions where the abundances of Fe II were independent on the equivalent widths or the rms scatter of the abundances becomes a minimum. Values for this species were derived using lines with $g f$ values from Martin et al. (1988) (MF values) and also with $g f$-values from compatible sources, in this case from Kurucz \& Bell (1995) (KX values). From FeII lines a mean microturbulence of $1.7 \mathrm{~km} \mathrm{~s}^{-1}$ is found for HD 206653 (Figs. 1 and 2). The CrII lines yield $1.4 \mathrm{~km} \mathrm{~s}^{-1}$ while the TiII lines suggest $0.0 \mathrm{~km} \mathrm{~s}^{-1}$. The difference between the values estimated from different species can be attributed to the fact that the silicon stars have magnetic fields. Thus, the Zeeman broadening is different from line to line. For weak lines it is a pseudomicroturbulence that combines in quadrature with any real microturbulence. A mean value of $1.7 \mathrm{~km} \mathrm{~s}^{-1}$ was finally adopted. We should point out that the above difference between Ti II and $\mathrm{Fe}$ II results have been observed also in our previous analysis of other CP stars.

According to the radiation diffusion scenario (Michaud \& Proffit 1993), the atmospheres of the mCP stars should be quiescent. Thus, the true microturbulence should be zero. However, when we examine the spectra of most mCP stars in an attempt to find microturbulence by standard techniques, we find non-zero values due to line broadening produced by the Zeeman effect acting as a pseudo-microturbulence. Following 


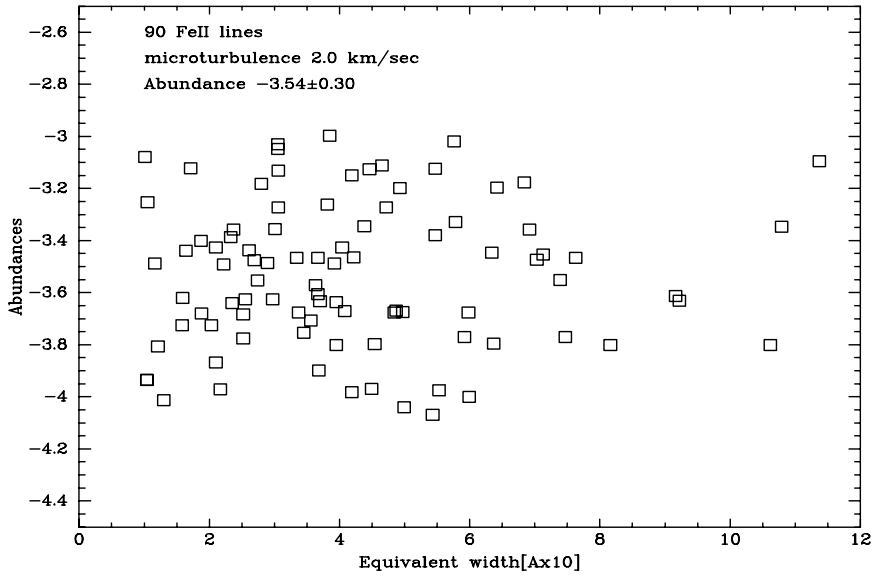

Fig. 1. Abundance vs. Equivalent width for 90 FeII lines, using the $g f$ values from Martin et al. (MF) plus Kurucz values (KX). The minimum slope and minimum scatter is obtained for a microturbulence of $2.0 \mathrm{~km} \mathrm{~s}^{-1}$.

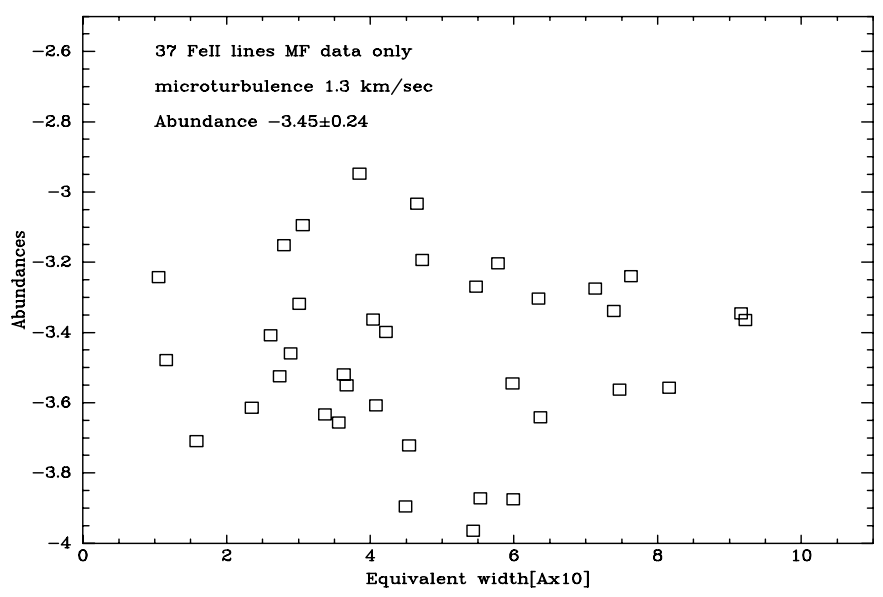

Fig. 2. Abundance vs. Equivalent width for 37 FeII lines, using only the $g f$ values from Martin et al. (MF). The minimum slope and minimum scatter is obtained for a microturbulence of $1.3 \mathrm{~km} \mathrm{~s}^{-1}$.

Adelman (1973) we equated the mean width of the Zeeman $\sigma$ components to the Doppler broadening to derive an effective microturbulence for each line that increases with the strength of the magnetic field and the width of the Zeeman pattern. With this method we can only have an approximate value of the magnetic field but it is adequate for the quality of our observational data. We have previous evidence of a magnetic field in HD 206653. North \& Cramer (1984) found a field of $1.5 \mathrm{kG}$. By assuming that there is no microturbulence and requiring that the abundances be independent of the derived magnetic field or that the scatter in the derived abundances be a minimum, we found from FeII lines that the surface magnetic field of HD 206653 (Figs. 3-6) is about $1.6 \mathrm{kG}$. This value agrees with the North \& Cramer (1984) determination. The CrII lines yield $1.7 \mathrm{kG}$ and the TiII lines $0.0 \mathrm{kG}$. As the rms values about the mean are similar for both methods, we present the final abundance results for the assumption of a uniform microturbulence of $1.7 \mathrm{~km} \mathrm{~s}^{-1}$ for HD 206653.

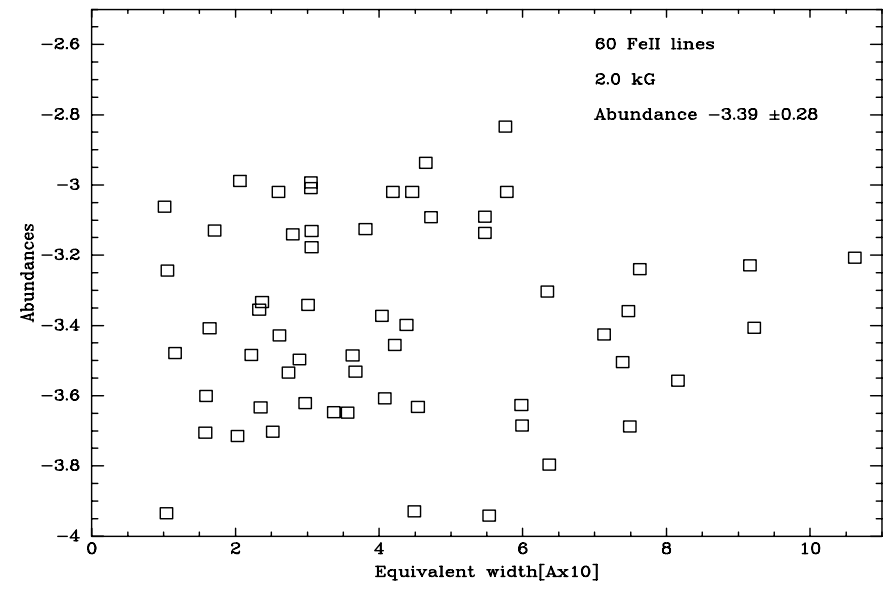

Fig. 3. Abundance vs. Equivalent width for $60 \mathrm{FeII}$ lines, using the $g f$ values from Martin et al. (MF) plus Kurucz values (KX). The minimum slope is obtained for a value of $2.0 \mathrm{kG}$.

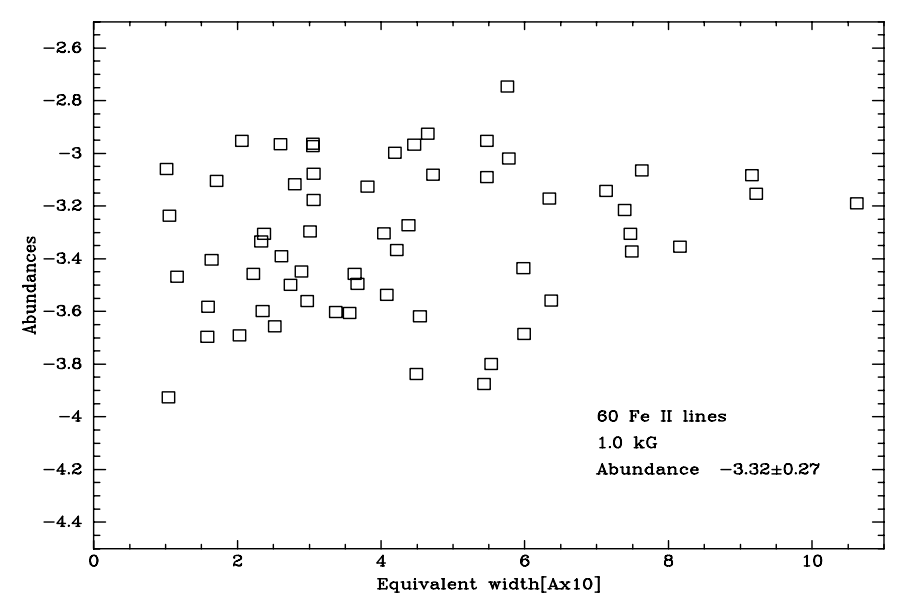

Fig. 4. Abundance vs. Equivalent width for 60 FeII lines, using the $g f$ values from Martin et al. (MF) plus Kurucz values (KX). The minimum scatter is obtained for a value of $1.0 \mathrm{kG}$.

\section{Discussion and conclusion}

Table 2 lists the derived abundances for HD 206653 for each atomic species. We have included the rms of the average abundance for each species, and between parenthesis we have indicated the number of lines involved on average. No highly blended lines were included. The table compares the abundances for HD 206653 with solar values (Grevesse et al. 1996) and also with the abundances of other Silicon stars, HD 43819 (López-García \& Adelman 1994), HD 133029 and HD 192913 (López-García \& Adelman 1999), HD 170973 (López-García et al. 2001). Compared to the sun, the light elements in the atmosphere of HD 206653 are solar except for Si, which is overabundant by a factor 5. Carbon seems to be highly deficient but its abundance is determined from only two lines. Al and S are represented by only one line. The "iron peak elements" are all overabundant, $\mathrm{Ti}, \mathrm{Cr}, \mathrm{Fe}$ and $\mathrm{Ni}$ by factors of $45,25,10$ and 4, respectively. Sr and Y are overabundant by a factor 1000 . For $\mathrm{Zr}$ and $\mathrm{Ba}$ we have only identified one line. Of the rare earths only $\mathrm{Ce}$ and $\mathrm{Eu}$ are present, while in the other silicon stars included for comparison in Table 2, we have identified 
Table 2. Comparison of derived and solar abundances.

\begin{tabular}{|c|c|c|c|c|c|c|}
\hline & HD 206653 & HD 170973 & HD 192913 & HD 133029 & HD 43819 & Sun \\
\hline$T_{\text {eff }}$ & 14385 & 10750 & 10900 & 11200 & 11300 & \\
\hline $\log g$ & 3.86 & $\mathrm{i} 3.50$ & 3.40 & 3.84 & 3.20 & \\
\hline Elements & $\log \mathrm{N} / \mathrm{H}$ & $\log \mathrm{N} / \mathrm{H}$ & $\log \mathrm{N} / \mathrm{H}$ & $\log \mathrm{N} / \mathrm{H}$ & $\log \mathrm{N} / \mathrm{H}$ & $\log \mathrm{N} / \mathrm{H}$ \\
\hline C II & $-4.70 \pm 0.04(4)$ & -3.73 & -3.16 & -3.08 & -3.78 & -3.45 \\
\hline $\mathrm{Mg}$ II & $-4.55 \pm 0.18(5)$ & -4.52 & -4.70 & -4.26 & -4.72 & -4.42 \\
\hline $\mathrm{Al} \mathrm{I}$ & $-4.20 * \pm 0.19(1)$ & -5.46 & -5.33 & $\ldots$ & $\ldots$ & -5.53 \\
\hline Si II & $-3.73 \pm 0.43(9)$ & -3.82 & -4.06 & -3.36 & -3.88 & -4.45 \\
\hline Si III & $-4.50 \pm 0.81(3)$ & -4.16 & $\ldots$ & $\ldots$ & $\ldots$ & -4.45 \\
\hline S II & $-5.52 *$ & -4.19 & -4.67 & -4.84 & -5.46 & -4.67 \\
\hline $\mathrm{Ca}$ II & $-5.91 *$ & -4.91 & -5.33 & -5.72 & -5.12 & -5.64 \\
\hline Ti II & $-5.33 \pm 0.34(25)$ & -5.11 & -5.60 & -6.02 & -5.81 & -6.98 \\
\hline Cr II & $-4.97 \pm 0.27(25)$ & -4.80 & -4.92 & -4.15 & -5.02 & -6.33 \\
\hline $\mathrm{Fe}$ II & $-3.51 \pm 0.30(98)$ & -3.50 & -3.36 & -3.28 & -3.66 & -4.50 \\
\hline $\mathrm{Fe}$ III & $-4.66^{*}$ & -2.92 : & $-2.68:$ & -3.18 & -3.70 & -4.50 \\
\hline Ni II & $-5.15 \pm 0.61(2)$ & -5.73 & -5.26 & -5.70 & -6.49 & -5.75 \\
\hline Sr II & $-5.80 \pm 0.27(4)$ & -5.74 & -5.03 & -7.01 & -5.63 & -9.03 \\
\hline Y II & $-6.60 *$ & -7.48 & -7.59 & -7.79 & $\ldots$ & -9.76 \\
\hline $\mathrm{Zr}$ II & $-5.71 * \pm 0.02$ & -6.98 & -7.22 & -7.53 & -7.81 & -9.40 \\
\hline Ba II & $-7.39 *$ & $\ldots$ & -8.52 & -8.68 & $\ldots$ & -9.87 \\
\hline Ce II & $-5.48 \pm 0.43(2)$ & -6.26 & -6.86 & -7.14 & -6.86 & -10.42 \\
\hline $\mathrm{Eu}$ II & $-6.17 \pm 0.52(2)$ & -6.27 & -5.29 & -8.44 & -8.15 & -11.49 \\
\hline $\mathrm{Hg}$ II & $-6.11 *$ & -4.39 & -5.12 & -6.57 & $\ldots$ & -10.83 \\
\hline
\end{tabular}

Note: * Indicates that the abundance is determined from one or two lines only.

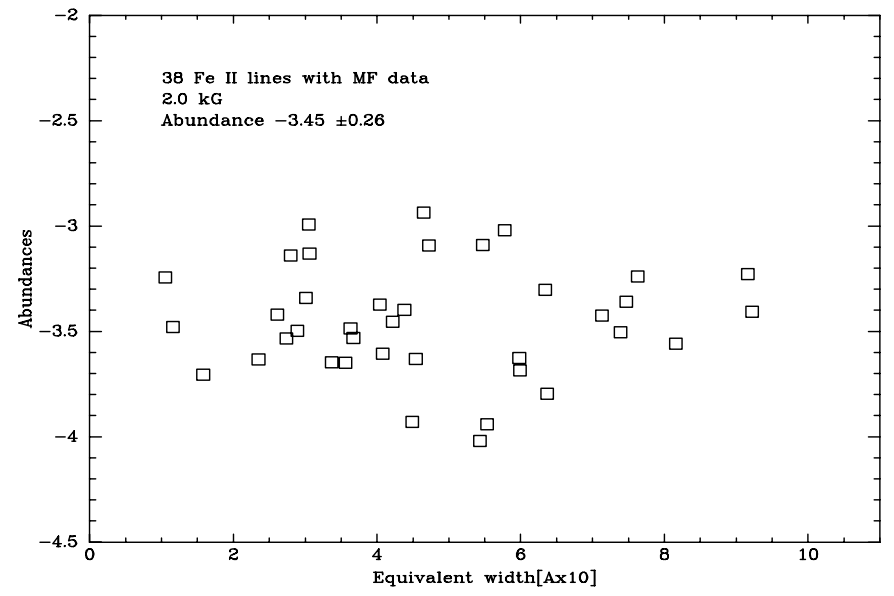

Fig. 5. Abundance vs. Equivalent width for 38 FeII lines, using only the $g f$ values from Martin et al. (MF). The minimum slope is obtained for a value of $2.0 \mathrm{kG}$.

also Pr, Nd, Sm, and Gd. For HD 192913 Dy also has been found. He I lines are not seen in the spectrum of HD 206653. Summarizing the results for HD 206653 indicates that it is a silicon star with characteristics similar to those found by other authors for this subclass of CP stars. The magnetic field has been determined in a crude mode, but the result agrees with a previous determination. A critical comparison with theory will be done once a larger and consistently analyzed sample becomes available.

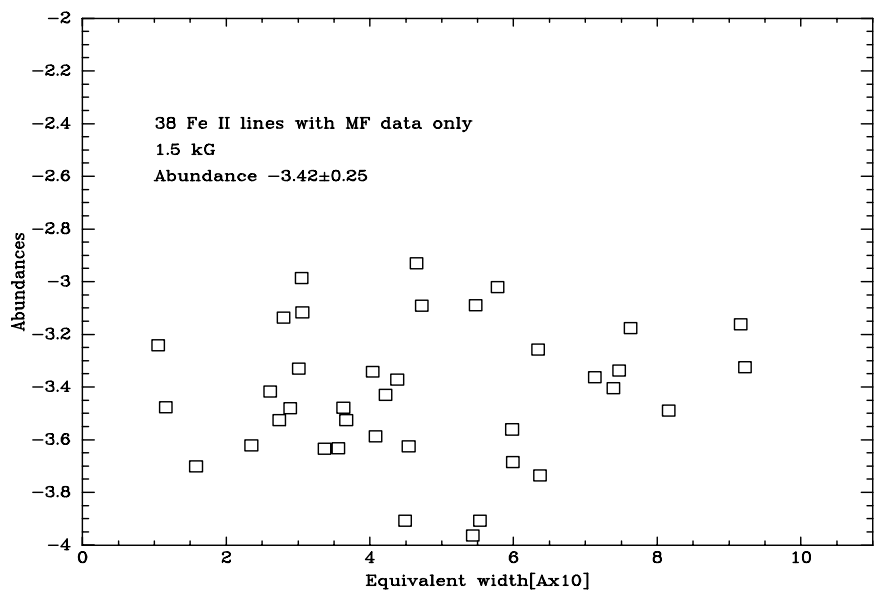

Fig. 6. Abundance vs. Equivalent width for 38 FeII lines, using only the $g f$ values from Martin et al. (MF). The minimum scatter is obtained for a value of $1.5 \mathrm{kG}$.

Acknowledgements. This work was partially supported through a grant from the Consejo Nacional de Investigaciones Científicas y Técnicas, República Argentina PIP 4279.

J. F. Albacete Colombo completed this work as a Fellow from the Agencia Nacional de Promoción Científica y Tecnológica (Grant 1505/3).

The CD and data aqcuisition system at CASLEO has been partly financed by R. M. Rich through U.S. NSF Grant AST-90-15827.

The authors acknowledge partial support by grants from FONCYT, number 03-1505 and ZLG and SM acknowledge support from CICITCA, UNSJ. We thank Saul Adelman for calculating the 
ATLAS model and for useful comments. We appreciate very much the comments of the referee, Dr. J. Zverko, that help to improve the presentation of the paper.

\section{References}

Adelman, S. J. 1973, ApJ, 183, 95

Adelman, S. J., \& Rayle, K. E. 2000, A\&A, 355, 308

Adelman, S. J., \& Pintado, O. I. 2000, A\&A, 354, 899

Adelman, S. J., \& Pintado, O. I. 1997, A\&A, 125, 219

Albacete Colombo, J. F., Lopez García, Z., Levato, H, Malaroda, S., \& Grosso, M. 2001, in preparation

Bidelman, W., \& Mac Connell, W. P. 1973, AJ, 78, 687

Chapelle, J., \& Sahal-Brechot, S. 1970, A\&A, 6, 415

Dworetsky, M. M. 1980, A\&A, 84, 350

Glagolevskij 1994, Bull Spec. Astrophys. Obs., 38, 152

Grevesse, N., Noels, A., \& Sauval, A. J. 1996, in Cosmic Abundances, ed. S. S. Holt, \& G. Sonneborn (ASP, San Francisco), ASP Conf. Ser., 99, 117

Hauck, B., \& Mermilliod, M. 1980, A\&AS, 40, 1

Hensberge, H., De Loore, C., Zuiderwijk, E. J., \& HammerschlagHensberge, G. 1977, A\&A, 54, 443

Huldt, S., Johansson, S., Litzen, U., \& Wyart, J.-F. 1982, Phys. Scr., 25,401

Iglesias, L., \& Velasco, R. 1964, Publ. Inst. Opt. Madrid, No. 23

Johansson, S. 1978, Phys. Scr., 18, 217

Kurucz, R. L. 1992, private communication

Kurucz, R. L., \& Bell, B. 1995, Kurucz CD-Rom No. 23, HarvardSmithsonian Center for Astrophysics, Smithsonian Astrophysical Observatory (Cambridge, MA)

Lanz, T. 1987, Thesis No. 2258, Geneva University

Lanz, T., Dimitrijevic, M. S., \& Artru, M.-C. 1988, A\&A, 192, 249
Levato, H., Malaroda, S., Morrell, N., Solivella, G., \& Grosso, M. 1996, A\&AS, 118, 231

López-García, Z., \& Adelman, S. J. 1994, A\&AS, 107, 353

López-García, Z., \& Adelman, S. J. 1999, A\&AS, 137, 227

López-García, Z., Adelman, S. J., \& Pintado, O. I. 2001, A\&A, 367, 859

Maitzen, H. M., Paunzen, E., Vogt, N., \& Weiss, W. W. 2000, A\&A, $355,1003 \mathrm{a}$

Maitzen, H. M., \& Vogt, N. 1983, A\&A, 123, 48

Martin, G. A., Fuhr, J. R., \& Wiese, W. L. 1988, J. Phys. Chem. Ref. Data, 1, Suppl. 3

Michaud, P., \& Proffit, C. R. 1993, in IAU Colloq. 137, ed. W. W. Weiss, \& A. Baglin, ASP Conf. Ser. 40 (San Francisco: ASP), 246

Moore, C. E. 1945, A Multiplet Table of Astrophysical Interest, Princeton University Observatory

Napiwotzki, R., Schonberner, D., \& Wenske, V. 1993, A\&A, 268, 653

North, P., \& Cramer 1984, A\&AS, 58, 387

North, P., \& Kroll, R. 1989, A\&AS, 78, 325

North, P., \& Nicolet, B. 1990, A\&A, 228, 78

Perryman, M. A. C., Lindegren, L., Kovalevsky, J., et al. 1997, A\&A, 323, L49

Pettersson, J. E. 1983, Phys. Scr., 28, 421

Pintado, O. I., \& Adelman, S. J. 1996, A\&AS, 118, 283

Pintado, O. I., Adelman, S. J., \& Gulliver, A. F. 1998, A\&AS, 129, 563

Piskunov, N. E. 1996, in Model Atmospheres and Spectrum Synthesis, ed. S. J. Adelman, F. Kupka, \& W. W. Weiss (ASP, San Francisco), ASP Conf. Ser., 108, 307

Reader, J., \& Corliss, C. H. 1980, NSRDS-NBS 68, Part 1, US Government Printing Office, Washington, DC

Sahal-Brechot, S. 1969, A\&A, 2, 322 